\title{
Electron cooling in probe collection from magnetized plasmas with anomalous transport
}

\author{
M Charro and J R Sanmartín
}

\begin{abstract}
The electron-retarding range of the current-voltage characteristic of a flat Langmuir probe perpendicular to a strong magnetic field in a fully ionized plasma is analysed allowing for anomalous (Bohm) cross-field transport and temperature changes in the collection process. With probe size and ion thermal gyroradius comparable, and smaller than the electron mean free path, there is an outer quasineutral region with ion viscosity determinant in allowing nonambipolar parallel and cross flow. A potential overshoot lying either at the base or inside the quasineutral region both makes ions follow Boltzmann's law at negative bias and extends the electron-retarding range to probe bias $e \phi_{\mathrm{P}} \sim+2 T_{\infty}$. Electron heating and cooling occur roughly at positive and negative bias, with a $T_{\mathrm{e}}$-minimum around $e \phi_{\mathrm{P}} \sim-2 T_{\infty}$; far from the probe heat conduction cools and heats electrons at and radially away from the probe axis, respectively. The potential overshoot with no thermal effects would reduce the electron current $I_{\mathrm{e}}$, making the $\ln I_{\mathrm{e}}$ versus $\phi_{\mathrm{P}}$ graph downwards-concave, but cooling further reduces $I_{\mathrm{e}}$ substantially, and may tilt the slope upwards past the temperature minimum. The domain of strict validity of our analysis is narrow in case of low ion mass (deuterium), breaking down with the ion Boltzmann law.
\end{abstract}

\section{Introduction}

In a strongly magnetized plasma, perturbations by an electron-collecting Langmuir probe reach far away, making charge transport essential to the workings of probes as particle sinks. The electron current $I_{\mathrm{e}}$ (and the current-voltage probe characteristic) may be affected, however, by energy and momentum, as well as particle, transport. A basic point rarely discussed in the literature concerns the issue of electron collection as an isothermal process $[1,2]$.

The variety of parameters involved in probe collection in the presence of a strong magnetic field $\bar{B}$ allow for quite different regimes. In cold but rarefied, unbounded collisionless space 
plasmas, probes may be biased highly positive [3], with recent theoretical developments for new applications $[4,5]$. Ionospheric experiments have found particles heated in the plasma beyond probe $[6,7]$ or spacecraft $[8]$ sheaths.

On the other hand, in hotter but denser bounded plasmas where collisional or turbulent transport applies, as in some large laboratory facilities [9] or at the edge of toroidal fusion machines, probe potential $\phi_{\mathrm{P}}$ might need to be highly negative to reduce the power flux reaching the probe. The floating potential and the ion branch of the $C-V$ characteristic are often made use of $[10,11]$, although such measurements involve problematic consideration of both tail of the electron distribution function, and ion-current saturation. Note further that the magnetic field itself will greatly reduce the power flux in the electron-retarding range of the characteristic ( $\phi_{\mathrm{P}}$ between floating and plasma potentials). The retarding range serves in determining the electron temperature $T_{\mathrm{e}}$, or for general double-probe use, and remains a basic problem in magnetized plasmas.

Bohm's pioneer work on probes in strongly magnetized plasmas was concerned with positive bias [12]. Broadly, generic results on the retarding range were first established by Sanmartin [13] using classical (collisional) cross-field transport (as against anomalous or turbulent transport in [12]): a potential overshoot along the flux tube terminating in the probe, ion density paradoxically following the Boltzmann law at $\phi_{\mathrm{P}}<0$, and the retarding range effectively extending up to a positive bias $\phi_{\mathrm{P}} \approx+(1-2) T_{\mathrm{e}} / e$. The potential hill result of the overshoot, which makes the $\ln I_{\mathrm{e}}-\phi_{\mathrm{P}}$ graph concave-downwards, has been experimentally detected in weakly ionized [14] and turbulent [15] plasmas, and may affect interpretation of data from multiple close probes. Free streaming rather than diffusive motion along the field, and hill effects on measurements, were discussed by Cohen [16]. Stangeby introduced the potential hill from [13] into a simplified form of Bohm's analysis and got a practical description of the retarding range and beyond [17]. We note that the collection process was considered isothermal in [13].

Recently, a fully consistent analysis of the standard single-probe allowing for thermal effects found electron cooling within certain range of probe size and bias [2]. The simplest probe model was adopted: a disc of radius $R$ perpendicular to an uniform magnetic field, and the complete set of macroscopic equations with classical transport coefficients as given by Braginskii [18]. Two points were missing from that work, however. First, cross-field transport is often anomalous; second, single probe theory, which requires current density to 'vanish' in the far plasma (effectively coming from large chamber walls in a laboratory), may not apply because distances to near walls are short [19].

Here, we further consider electron cooling and heating processes for the retarding range of a probe, ascertaining their generic character and domain of validity, and allowing for anomalous cross-field diffusion. Anomalous transport is a deep and unsettled field of work but transport in the edge plasma has become crucial to magnetic confinement [20]; Langmuir probes are regularly used in toroidal machines, both in the scrape-off layer and inside the separatrix, and extensive work has been carried out on the physics of fluctuations, on overall modelling of the edge plasma, and in refining probe measurements [21-23]. Note, anyhow, that transport to a probe differs from global toroidal transport, where you might reasonably use flux-surface averages for simple analyses: averaging over the 'toroidal' length would here miss the basic fact that transport processes end at a limiting sheath, which additionally acts as a differential-particle sink. In case of fusion plasmas our transport model may be called local: we take parallel transport fully classical rather than neoclassical; cross-field diffusion of Bohm rather than Kadomtsev's gyro-Bohm type (which essentially replaces mean free path by minor plasma radius in classical cross-field diffusion) [24]; and the welldefined structure of collisional theory [18] as regards off-diagonal terms of a transport matrix 
(bootstrap current, Ware pinch convection...) [25]. There have been early attempts at using anomalous transport in electron collection [26]. There naturally remain uncertainties in the transport description.

Only the electron current will be considered. Ignoring the ion current will clearly fail around and below the floating potential; this precludes directly applying our analysis for double-probe use, say, for a flush-mounted (adjacent double) probe [11,27]. Double-probe modelling will be the subject of following work. Our probe would be a protruding (maybe reciprocating) probe [28]. The possibility of ionization and other kinetic effects from neutrals, or flow in the unperturbed plasma, will be ignored here. Analyses of parallel flow for the ion branch of the characteristic has given rise to so-called Mach probes [29, 30]. Perpendicular flow, whether related to static or fluctuating electric fields, has been analysed recently [10]. We note that ionospheric experiments suggest that spacecraft velocity, even though highly subsonic as regards electrons, might have a substantial effect on electron collection at highly positive bias $[7,31,32]$.

For simplicity, we take ion charge $Z=1$, and equal unperturbed temperatures, $T_{1 \infty}=T_{\mathrm{e} \infty} \equiv T_{\infty}$. We assume very large $\Omega_{\mathrm{e}} \tau_{\mathrm{e} \infty}$, where $\Omega_{\mathrm{e}}\left(\equiv e B / m_{\mathrm{e}}\right)$ and $\tau_{\mathrm{e}}$ are electron gyrofrequency and Braginskii's collision time, respectively; we will write $\Omega_{\mathrm{e}} \tau_{\mathrm{e} \infty} \equiv \lambda_{\infty} / l_{\mathrm{e} \infty}$, with electron thermal gyroradius $l_{\mathrm{e}} \equiv c_{\mathrm{e}} / \Omega_{\mathrm{e}}$, characteristic mean free path $\lambda \equiv c_{\mathrm{e}} \tau_{\mathrm{e}}$, and thermal velocity $c_{\mathrm{e}} \equiv \sqrt{ } T_{\mathrm{e}} / m_{\mathrm{e}}$. Our analysis involves a bias ratio, $e \phi_{\mathrm{P}} / T_{\infty}$ (in the range -3 to +1 , say), and three large length ratios, $\lambda_{\infty} / l_{\mathrm{e} \infty}, R / l_{\mathrm{e} \infty}$, and $l_{1 \infty} / l_{\mathrm{e} \infty}\left(\equiv \sqrt{ } m_{1} / m_{\mathrm{e}}\right)$. We take $R \sim l_{1 \infty}$ and $\lambda_{\infty} / l_{1 \infty}$ about unity or moderately large. No Debye sheath analysis will be required. Although no ion transport term will have direct quantitative effect on the results, ion viscosity proves relevant in sustaining both parallel and cross-field non-ambipolar quasineutral flow, a fact that has produced some confusion in the past [33].

The collisional study of [2] is recalled in section 2. Anomalous cross-field transport is consistently integrated into a full model for probe collection in section 3. Detailed graphical results are presented and discussed in section 4 . The domain of validity of the model is discussed in section 5. Results are resumed in section 6.

\section{Fully classical transport}

Here, we briefly recall the fully steady, collisional transport case [2]. Taking (i) electron velocities well below sonic (to ignore inertia terms) and (ii) electron viscosity effects negligible, as conditions to verify below, the electron momentum equations read

$$
\begin{aligned}
& 0 \approx-\frac{\partial p_{\mathrm{e}}}{\partial z}+e n \frac{\partial \phi}{\partial z}+R_{\mathrm{e} z}, \\
& 0 \approx e B n v_{\mathrm{e} r}+R_{\mathrm{e} \theta} \\
& 0 \approx-\frac{\partial p_{\mathrm{e}}}{\partial r}+e n \frac{\partial \phi}{\partial r}-e B n v_{\mathrm{e} \theta}+R_{\mathrm{e} r} \quad\left(R_{\mathrm{e} r} \text { negligible }\right),
\end{aligned}
$$

$\bar{R}_{\mathrm{e}}$ is the force on electrons due to collisions with ions, the magnetic field lies along the $z$-axis of cylindrical coordinates $(\partial / \partial \theta \equiv 0)$, and the plasma is quasineutral outside a thin sheath $\left(n_{1} \approx n_{\mathrm{e}} \equiv n\right)$. Under a third ansatz, (iii) $\bar{v}_{\mathrm{e}}-\bar{v}_{1} \approx \bar{v}_{\mathrm{e}}$, one finds

$$
\begin{aligned}
& R_{\mathrm{e} z} \approx-\alpha_{0} \frac{m_{\mathrm{e}}}{\tau_{\mathrm{e}}} n v_{\mathrm{e} z}-\beta_{0} n \frac{\partial T_{\mathrm{e}}}{\partial z}, \\
& R_{\mathrm{e} \theta} \approx-\frac{m_{\mathrm{e}}}{\tau_{\mathrm{e}}} n v_{\mathrm{e} \theta}-\frac{\beta_{1}^{\prime \prime}}{\Omega_{\mathrm{e}} \tau_{\mathrm{e}}} n \frac{\partial T_{\mathrm{e}}}{\partial r},
\end{aligned}
$$


with Braginskii constants $\alpha_{0}, \beta_{0}, \beta_{1}^{\prime \prime}$, while $R_{\mathrm{er}}$ is found to be smaller than dominant terms in (2) by a factor $1 / \Omega_{\mathrm{e}}^{2} \tau_{\mathrm{e}}^{2} \equiv l_{\mathrm{e}}^{2} / \lambda^{2}$.

Using $n v_{\mathrm{e} z}$ and $n v_{\mathrm{e} r}$ from equations (1)-(3) in the electron continuity equation,

$$
\frac{\partial}{\partial z} n v_{\mathrm{e} z}+\frac{1}{r} \frac{\partial}{\partial r} r n v_{\mathrm{e} r}=0
$$

there results a first relation for $\phi, n$, and $T_{\mathrm{e}}$. Further, with $e \phi \sim T_{\mathrm{e}}$ and $r \sim R$, the characteristic values for length along $z$ and for velocity components come out to be

$$
\begin{aligned}
& L_{z} \sim R \Omega_{\mathrm{e}} \tau_{\mathrm{e}}=\frac{R \lambda}{l_{\mathrm{e}}}, \\
& \frac{v_{\mathrm{e} z}}{c_{\mathrm{e}}} \sim \frac{v_{\mathrm{e} \theta}}{c_{\mathrm{e}}} \sim \frac{l_{\mathrm{e}}}{R}, \\
& \frac{v_{\mathrm{e} r}}{c_{\mathrm{e}}} \approx \frac{l_{\mathrm{e}}^{2}}{\lambda R},
\end{aligned}
$$

showing $L_{z}$ large compared to both $R$ and $\lambda$, and verifying ansatz (i). As regards ansatz (ii), the electron viscous force, involving five viscous coefficients, $\eta_{\mathrm{e} 0-4}\left(\eta_{\mathrm{e} 1,2} \times \Omega_{\mathrm{e}}^{2} \tau_{\mathrm{e}}^{2} \sim\right.$ $\eta_{\mathrm{e} 3-4} \times \Omega_{\mathrm{e}} \tau_{\mathrm{e}} \sim \eta_{\mathrm{e} 0} \sim n T_{\mathrm{e}} \tau_{\mathrm{e}}$ ), is found to have components $F_{\mathrm{e} z}^{\mathrm{v}}, F_{\mathrm{e} \theta}^{\mathrm{v}}$, and $F_{\mathrm{e} r}^{\mathrm{v}}$ that are indeed smaller than $R_{\mathrm{e} z}, R_{\mathrm{e} \theta}$, and $e B n v_{\mathrm{e} \theta}$, respectively, by a factor of order $l_{\mathrm{e}}^{2} / R^{2}$.

The energy equation for electrons now takes the form

$$
\nabla \cdot\left[\left(\frac{5}{2} T_{\mathrm{e}}-e \phi\right) n \bar{v}_{\mathrm{e}}+\bar{q}_{\mathrm{e}}\right] \approx-Q_{1}+\bar{R}_{\mathrm{e}} \cdot \bar{v}_{1} \approx-Q_{1} \equiv-\frac{3 m_{\mathrm{e}}}{m_{1}} n \frac{T_{\mathrm{e}}-T_{1}}{\tau_{\mathrm{e}}},
$$

with $q_{\mathrm{e} z}$ and $q_{\mathrm{e} r}$ given by Braginskii,

$$
\begin{aligned}
& q_{\mathrm{e} z} \approx-T_{\mathrm{e}}\left[\gamma_{0} \frac{\tau_{\mathrm{e}}}{m_{\mathrm{e}}} n \frac{\partial T_{\mathrm{e}}}{\partial z}-\beta_{0} n v_{\mathrm{e} z}\right], \\
& q_{\mathrm{e} r} \approx-\frac{T_{\mathrm{e}}}{\Omega_{\mathrm{e}} \tau_{\mathrm{e}}}\left[\frac{\gamma_{1}^{\prime}}{e B} n \frac{\partial T_{\mathrm{e}}}{\partial r}+\beta_{1}^{\prime \prime} n v_{\mathrm{e} \theta}\right] .
\end{aligned}
$$

Note that all terms on the left-hand side of (6) are of order of $\bar{R}_{\mathrm{e}} \cdot \bar{v}_{\mathrm{e}}$, which ansatz (iii) makes much larger than $\bar{R}_{\mathrm{e}} \cdot \bar{v}_{1}$, whereas $Q_{1}$ is of order of $\bar{R}_{\mathrm{e}} \cdot \bar{v}_{\mathrm{e}} \times 3 R^{2} / l_{1}^{2}$ and might be comparable to $\bar{R}_{\mathrm{e}} \cdot \bar{v}_{\mathrm{e}}$. Equation (6) provides a second relation among $\phi, n, T_{\mathrm{e}}$ and $T_{1}$.

Next, assuming (iv) ion velocities well below sonic too, and using ansatz (iii) to ignore any magnetic force component, the ion $z$ - and $\theta$-momentum equations read

$$
\begin{aligned}
& 0 \approx-\frac{\partial p_{1}}{\partial z}-e n \frac{\partial \phi}{\partial z}+F_{1 z}^{\mathrm{v}}-R_{\mathrm{e} z}, \\
& 0 \approx F_{1 \theta}^{\mathrm{v}}-R_{\mathrm{e} \theta},
\end{aligned}
$$

where viscous forces, to order $\sqrt{m_{\mathrm{e}} / m_{1}}$, read

$$
\begin{aligned}
& F_{1 z}^{\mathrm{v}} \approx \frac{1}{r} \frac{\partial}{\partial r}\left(r \eta_{12} \frac{\partial v_{1 z}}{\partial r}\right), \\
& F_{1 \theta}^{\mathrm{v}} \approx\left[\frac{1}{r} \frac{\partial}{\partial r}\left(r \eta_{11} \frac{\partial}{\partial r}\right)-\frac{\eta_{11}}{r^{2}}\right] v_{1 \theta},
\end{aligned}
$$




$$
\begin{array}{ll}
\frac{\Omega_{1}^{2} \tau_{1} \eta_{11}}{n T_{1}} \approx 0.2(0.3) & \text { for } \Omega_{1}^{2} \tau_{1}^{2}=1(\gg 1), \\
\frac{\Omega_{1}^{2} \tau_{1} \eta_{12}}{n T_{1}} \approx 0.47(1.2) & \text { for } \Omega_{1}^{2} \tau_{1}^{2}=1(\gg 1) ;
\end{array}
$$

the last term in $(9 b)$ was overlooked in [2], with no effect, however, in estimating $v_{1 \theta}$. Equations (8) and (9) yield $v_{1 z}$ and $v_{1 \theta}$, the ion continuity equation

$$
\frac{\partial}{\partial z} n v_{1 z}+\frac{1}{r} \frac{\partial}{\partial r} r n v_{1 r}=0
$$

then giving $v_{1 r}$. This allows verifying ansatzen (iii) and (iv),

$$
\begin{aligned}
\frac{v_{1 z}}{v_{\mathrm{e} z}} \sim \frac{v_{1 \theta}}{v_{\mathrm{e} \theta}} \sim \frac{v_{1 r}}{v_{\mathrm{e} r}} \sim \frac{R^{2}}{l_{1}^{2}} \sqrt{\frac{m_{\mathrm{e}}}{m_{1}}}, \\
\frac{v_{1 z}}{c_{1}} \sim \frac{v_{1 \theta}}{c_{1}} \sim \frac{R}{l_{1}} \sqrt{\frac{m_{\mathrm{e}}}{m_{1}}}, \\
\frac{v_{1 r}}{c_{1}} \approx \frac{R}{\lambda} \frac{m_{\mathrm{e}}}{m_{1}} .
\end{aligned}
$$

Finally, the radial flux term of the heat-flux divergence in the ion energy equation is larger than $Q_{1}$ by a factor $\left(m_{1} / m_{\mathrm{e}}\right)^{1 / 2} \times l_{1}^{2} / 3 R^{2}, Q_{1}$ itself being dominant against all other terms in the equation, which thus reads

$$
\frac{1}{r} \frac{\partial}{\partial r} r q_{1 r} \approx 0 \quad\left(q_{1 r} \propto \frac{\partial T_{1}}{\partial r}\right) .
$$

With $r q_{1 r}=\left.r q_{1 r}\right|_{r=0}=0$, at any $z$, the resulting equation, $\partial T_{1} / \partial r=0$, yields $T_{1}=T_{1}(z$, $r \rightarrow \infty)=T_{\infty}$. The ion $r$-momentum equation then reads

$0 \approx-T_{\infty} \frac{\partial n}{\partial r}-e n \frac{\partial \phi}{\partial r}+F_{1 r}^{\mathrm{v}}, \quad\left(F_{1 r}^{\mathrm{v}} \approx \frac{1}{r} \frac{\partial}{\partial r} r \eta_{13} \frac{\partial v_{1 \theta}}{\partial r}-\frac{\eta_{13} v_{1 \theta}}{r^{2}}\right.$ negligible $)$,

with $\Omega_{1} \eta_{13} / n T_{1} \approx 0.37(0.5)$ for $\Omega_{1}^{2} \tau_{1}^{2}=1(\gg 1)$, the viscous force $F_{1 r}^{\mathrm{v}}$ is smaller than the other terms by a factor $\sqrt{m_{\mathrm{e}} / m_{1}}$. Equation (14) gives $\ln n+e \phi / T_{\infty}=\ln n(z, r \rightarrow \infty)+$ $e \phi(z, r \rightarrow \infty) / T_{\infty}=\ln n_{\infty}$. Using results

$$
\begin{aligned}
& T_{1}=T_{\infty}, \\
& n=n_{\infty} \exp \left(-\frac{e \phi}{T_{\infty}}\right),
\end{aligned}
$$

in equations (4) and (6) provides two equations for $T_{\mathrm{e}}$ and $\phi$, which were analysed in [2].

Note how quasineutral diffusion comes out non-ambipolar, ion viscosity, along with two separate continuity equations, being crucial in this respect. The addition of equations ( $1 a$ ) and (8a) ( $z$-momentum equation for the ion-electron fluid) shows $F_{1 z}^{\mathrm{v}}$, and thus $v_{1 z}$, being driven by $z$-gradients, which also drive $R_{\mathrm{e} z}$, and thus $v_{\mathrm{e} z}$. For $R / l_{1}$ not large, (12a) proves $v_{1 z} / v_{\mathrm{e} z}$ small. The continuity equations (4) and (11) then yield a radial flux ratio small too, $v_{1 r} / v_{\mathrm{er}} \sim v_{1 z} / v_{\mathrm{e} z}$. Radial gradients drive $v_{\mathrm{e} \theta}$ in (2), and thus $R_{\mathrm{e} \theta}$, which drives both $v_{\mathrm{e} r}$ and $F_{1 \theta}^{\mathrm{v}}\left(v_{1 \theta}\right)$, leading to $v_{1 \theta} / v_{\mathrm{e} \theta}$ again small in $(12 a)$. 


\section{Anomalous cross-field transport}

The result for cross-field transport of electrons from equations (1b), (2) and (3b) reads

$$
e B n v_{\mathrm{e} r}=\frac{-1}{\Omega_{\mathrm{e}} \tau_{\mathrm{e}}}\left[\frac{\partial p_{\mathrm{e}}}{\partial r}-e n \frac{\partial \phi}{\partial r}-\beta_{1}^{\prime \prime} n \frac{\partial T_{\mathrm{e}}}{\partial r}\right] .
$$

For isothermal electrons and no electric field, equation (16) reads $n v_{\mathrm{e} r}=-D_{\mathrm{e} \perp} \times \partial n / \partial r$, with the classical diffusion coefficient $D_{\mathrm{e} \perp}=c_{\mathrm{e}}^{2} / \Omega_{\mathrm{e}}^{2} \tau_{\mathrm{e}}$. The replacement of $1 / \Omega_{\mathrm{e}} \tau_{\mathrm{e}} \equiv l_{\mathrm{e}} / \lambda$ in (16) by a numerical factor $\varepsilon$ describes Bohm cross-field transport, involving correlated fluctuations of density and electric field (and temperature and magnetic-field) that break the cylindrical symmetry and drive the averaged momentum equation; the diffusion coefficient is then $D_{\mathrm{e} B}=\varepsilon c_{\mathrm{e}}^{2} / \Omega_{\mathrm{e}}$. We may now check whether the solution of the previous section is consistent with a scale-up in electron cross-field diffusion by the large factor $\varepsilon \Omega_{\mathrm{e}} \tau_{\mathrm{e}} \equiv \varepsilon \lambda / l_{\mathrm{e}}$ $\left(\lambda / l_{\mathrm{e}}\right.$ very large, $\varepsilon$ small or moderately small, Bohm's suggested value being $\varepsilon=\frac{1}{16}$ [12]).

Electron momentum and heat cross-field transport should scale up by actor of the same order. Electron viscosity terms, which were of order $l_{\mathrm{e}}^{2} / R^{2}$ against dominant terms, remain negligible, however. This applies to the force component $R_{r}$ too, leaving $v_{\mathrm{e} \theta}$ unchanged in (2). With $R_{\mathrm{e} z}$ still given by equation (3a), and with $r \sim R$, equations (1a) and (4) show both $v_{\mathrm{e} z}$ and $1 / L_{z}$ greater by the factor $\sqrt{\varepsilon \lambda / l_{\mathrm{e}}}$. This yields velocity components

$$
\begin{aligned}
& \frac{v_{\mathrm{e} \theta}}{c_{\mathrm{e}}} \sim \frac{l_{\mathrm{e}}}{R}, \\
& \frac{v_{\mathrm{e} z}}{c_{\mathrm{e}}} \sim \frac{\sqrt{\varepsilon \lambda l_{\mathrm{e}}}}{R}, \\
& \frac{v_{\mathrm{e} r}}{c_{\mathrm{e}}} \sim \varepsilon \frac{l_{\mathrm{e}}}{R} .
\end{aligned}
$$

As regards equation (6) note that $q_{\mathrm{e} r}$, in fact the entire left-hand side, is now larger by the factor $\varepsilon \lambda / l_{\mathrm{e}}$, whereas $Q_{1}$ remains as given, thus becoming of order $R^{2} / \varepsilon \lambda l_{\mathrm{e}} \times 3 m_{\mathrm{e}} / m_{1}$ relative to the left-hand side; we are here assuming that fluctuations are slow (frequencies small against $\left.1 / \tau_{\mathrm{e}} \sim \Omega_{1} \times \sqrt{ } m_{1} / m_{\mathrm{e}} \times l_{1} / \lambda\right)$.

Although ions and electrons have similar Bohm diffusion coefficients $\left(c_{1}^{2} / \Omega_{1} \sim c_{\mathrm{e}}^{2} / \Omega_{\mathrm{e}}\right)$, replacing classical cross-field transport with Bohm transport rests on a more stringent condition in the case of ions $\left(\varepsilon \Omega_{1} \tau_{1} \sim \varepsilon \lambda / l_{1}\right.$ should be large). We shall now assume that $l_{1} / \lambda$ is larger than $\varepsilon$, ion viscosity coefficients thus retaining order of magnitude values as given in section 2 , allowing us to write $\eta_{11,2} \sim\left(n T_{1} / \Omega_{1}\right) \times l_{1} / \lambda, \eta_{13} \sim n T_{1} / \Omega_{1}$. Note, anyhow, that ion transport terms are only used to check consistency of the solution, not to determine it. Equations $(8 a)$, (9a) and (11) then yield

$$
\begin{aligned}
& \frac{v_{1 z}}{v_{\mathrm{e} z}} \sim \frac{v_{1 r}}{v_{\mathrm{e} r}} \sim \frac{R^{2}}{l_{1}^{2}} \sqrt{\frac{m_{\mathrm{e}}}{m_{1}}}, \\
& \frac{v_{1 z}}{c_{1}} \sim \frac{R}{l_{1}} \sqrt{\frac{\varepsilon \lambda}{l_{1}} \sqrt[4]{\frac{m_{\mathrm{e}}}{m_{1}}}} \\
& \frac{v_{1 r}}{c_{1}} \sim \frac{R}{l_{1}} \varepsilon \sqrt{\frac{m_{\mathrm{e}}}{m_{1}}} .
\end{aligned}
$$

Finally, with the radial ion heat-flux keeping its order of magnitude value while $Q_{1}$ remains as given, equation (14), leading to $T_{1}=T_{\infty}$, remains valid. All ratios in $(17 a)-(17 c),(18 a)-(18 c)$ are still small. 
Regarding $v_{1 \theta}$ we shall just assume now and in section 4 that the ion $\theta$-momentum equation, previously given by $(8 b)$, yields $v_{1 \theta}$ low enough to allow writing the $r$-momentum equation as in (14), leading to the ion Boltzmann law that is basic to our analysis. We shall determine conditions for the above result to apply in section 5, where we also discuss effects arising when it does not apply, and the general validity of our model. Here, use of (15a) and (15b) in equations (4) and (6) again yields two equations determining $\phi$ and $T_{\mathrm{e}}$, with $v_{\mathrm{e} r}$ and $q_{\mathrm{e} r}$ properly modified in (7b) and (16).

A model for anomalous transport with the full scope of the classical collisional description is lacking. For definiteness, we shall keep the brackets in (7b) and (16) after replacing $1 / \Omega_{\mathrm{e}} \tau_{\mathrm{e}}$ by $\varepsilon$, and use Braginskii's $\gamma_{1}^{\prime}$ coefficient. The (moderately) large value $\gamma_{1}^{\prime} \approx 4.664$ reflects the fact that superthermal electrons carry the heat flux [34], in agreement with suggestions that anomalous cross-field thermal diffusivity is large compared with corresponding particle diffusivity. As regards $\beta_{1}^{\prime \prime}$ we shall consider both Braginskii's value $\left(\frac{3}{2}\right)$ and value $\beta_{1}^{\prime \prime}=0$ (no thermoelectric effect). Introducing dimensionless variables,

$\tilde{r} \equiv \frac{r}{R}, \quad \tilde{z} \equiv \frac{z}{L_{z}}\left[L_{z} \equiv \frac{R}{\sqrt{\alpha_{0}}} \sqrt{\frac{\lambda_{\infty}}{\varepsilon l_{\mathrm{e} \infty}}}\right], \quad \tilde{\phi} \equiv \frac{e \phi}{T_{\infty}}=-\ln \frac{n}{n_{\infty}}, \quad \tilde{T} \equiv \frac{T_{\mathrm{e}}}{T_{\infty}}$,

equations (4) and (6) read

$$
\begin{gathered}
\frac{\partial}{\partial \tilde{z}}\left[\tilde{T}^{3 / 2}\left\{(1+\tilde{T}) \frac{\partial \tilde{\phi}}{\partial \tilde{z}}-\left(1+\beta_{0}\right) \frac{\partial \tilde{T}}{\partial \tilde{z}}\right\}\right] \\
+\frac{1}{\tilde{r}} \frac{\partial}{\partial \tilde{r}}\left[\tilde{r} \exp (-\tilde{\phi})\left\{(1+\tilde{T}) \frac{\partial \tilde{\phi}}{\partial \tilde{r}}+\left(\beta_{1}^{\prime \prime}-1\right) \frac{\partial \tilde{T}}{\partial \tilde{r}}\right\}\right]=0 \\
\frac{\partial}{\partial \tilde{z}} \tilde{T}^{3 / 2}\left[(1+\tilde{T})\left\{\left(\frac{5}{2}+\beta_{0}\right) \tilde{T}-\tilde{\phi}\right\} \frac{\partial \tilde{\phi}}{\partial \tilde{z}}-\left\{\left(\alpha_{0} \gamma_{0}+\frac{5}{2}+\frac{7}{2} \beta_{0}+\beta_{0}^{2}\right) \tilde{T}-\left(1+\beta_{0}\right) \tilde{\phi}\right\} \frac{\partial \tilde{T}}{\partial \tilde{z}}\right] \\
+\frac{1}{\tilde{r}} \frac{\partial}{\partial \tilde{r}} \tilde{r} \exp (-\tilde{\phi})\left[(1+\tilde{T})\left\{\left(\frac{5}{2}-\beta_{1}^{\prime \prime}\right) \tilde{T}-\tilde{\phi}\right\} \frac{\partial \tilde{\phi}}{\partial \tilde{r}}\right. \\
+ \\
\left.\left.+\left(\frac{7}{2} \beta_{1}^{\prime \prime}-\gamma_{1}^{\prime}-\frac{5}{2}\right) \tilde{T}-\left(\beta_{1}^{\prime \prime}-1\right) \tilde{\phi}\right\} \frac{\partial \tilde{T}}{\partial \tilde{r}}\right] \\
=-\frac{3 m_{\mathrm{e}}}{m_{1}} \frac{R^{2}}{\varepsilon \lambda_{\infty} l_{\mathrm{e}_{\infty}}} \times \frac{\exp (-2 \tilde{\phi})}{\tilde{T}^{3 / 2}}(\tilde{T}-1) .
\end{gathered}
$$

Boundary conditions

$$
\begin{array}{ll}
\frac{\partial \tilde{\phi}}{\partial \tilde{r}}=\frac{\partial \tilde{T}}{\partial \tilde{r}}=0 & \text { at } \tilde{r}=0, \\
\tilde{\phi} \rightarrow 0, \tilde{T} \rightarrow 1 & \text { as } \sqrt{\tilde{z}^{2}+\tilde{r}^{2}} \rightarrow \infty,
\end{array}
$$

and

$$
\frac{\partial \tilde{\phi}}{\partial \tilde{z}}=\frac{\partial \tilde{T}}{\partial \tilde{z}}=0 \quad \text { at } \tilde{z}=0, \tilde{r}>1,
$$

are manifest but boundary conditions for $\tilde{z} \rightarrow 0, \tilde{r}<1$ require detailed consideration. Equations $\left(4^{\prime}\right)$ and $\left(6^{\prime}\right)$ describe an outer collisional, quasineutral flow with ions following a Boltzmann law. These conditions break down at small enough $\tilde{z}$ values, covering an inner 
region where collisionality and the Boltzmann law for ions fail at $z \sim$ local mean free path and probe radius $\left(\ll L_{z}\right.$ ), while quasineutrality fails in an embedded sheath at $z \sim$ local Debye length. Throughout this overall inner region, where z-gradients are comparatively steep, equation (4) yields $n v_{\mathrm{e} z}=$ const. Using subscript 0 for outer solution values at $\tilde{r}<1, \tilde{z} \rightarrow 0$, we find that there is a bias range for which $\exp \left[e\left(\phi_{0}-\phi_{\mathrm{P}}\right) / T_{\mathrm{e} 0}\right]$ is large, electrons that come into the inner region thus facing a tall energy hill. The probe surface, even though absorbing, acts as a perfectly reflecting wall for most of the electron distribution function, which approaches the inner region as the slightly distorted Maxwellian of Braginskii's calculations but reaches the probe as a (truncated) Maxwell-Boltzmann distribution at values $n_{0}, T_{0}$. A detailed discussion of the inner region is given in [2].

We can now determine the constant value of $n v_{\mathrm{e} z}$ by evaluating it at the probe, to get

$n_{0} v_{\mathrm{e} z 0}=-n_{0} \exp \left[-\frac{e\left(\phi_{0}-\phi_{\mathrm{P}}\right)}{T_{\mathrm{e} 0}}\right] \times \sqrt{\frac{T_{\mathrm{e} 0}}{2 \pi m_{\mathrm{e}}}}, \quad\left[n_{0}=n_{\infty} \exp \left(-\frac{e \phi_{0}}{T_{\infty}}\right)\right]$.

The total energy flux along $z$ on the left-hand side of equation (6) is similarly conserved, yielding

$$
\left(\frac{5}{2} T_{\mathrm{e} 0}-e \phi_{0}\right) n_{0} v_{\mathrm{e} z 0}+q_{\mathrm{e} z 0}=\left(2 T_{\mathrm{e} 0}-e \phi_{\mathrm{P}}\right) n_{0} v_{\mathrm{e} z 0}
$$

In dimensionless variables, using results for $n v_{\mathrm{e} z}$ and $q_{\mathrm{e} z}$ from section 2 , these equations read

$$
\begin{aligned}
& \tilde{T}_{0}\left[\left.\left(1+\beta_{0}\right) \frac{\partial \tilde{T}}{\partial \tilde{z}}\right|_{0}-\left.\left(1+\tilde{T}_{0}\right) \frac{\partial \tilde{\phi}}{\partial \tilde{z}}\right|_{0}\right]=\sqrt{\frac{\alpha_{0}}{2 \pi}} \times \frac{R}{\sqrt{\varepsilon \lambda_{\infty} l_{\mathrm{e} \infty}}} \exp \left[\frac{e \phi_{\mathrm{P}}}{T_{\infty} \tilde{T}_{0}}-\frac{\tilde{T}_{0}+1}{\tilde{T}_{0}} \tilde{\phi}_{0}\right], \\
& {\left[\left.\left(1+\beta_{0}\right) \frac{\partial \tilde{T}}{\partial \tilde{z}}\right|_{0}-\left.\left(1+\tilde{T}_{0}\right) \frac{\partial \tilde{\phi}}{\partial \tilde{z}}\right|_{0}\right]\left[\frac{\tilde{\phi}_{0}-e \phi_{\mathrm{P}} / T_{\infty}}{\tilde{T}_{0}}-\beta_{0}-\frac{1}{2}\right]=\alpha_{0} \gamma_{0} \frac{\partial \tilde{T}}{\partial \tilde{z}} .}
\end{aligned}
$$

Coupled equations $\left(4^{\prime}\right)$ and $\left(6^{\prime}\right)$, with boundary conditions $\left(20^{\prime}\right)$ and $\left(21^{\prime}\right)$, serve to determine $\tilde{\phi}$ and $\tilde{T}$, and finally the current reaching the probe. The full set of equations involves just three dimensionless parameters, $e \phi_{\mathrm{P}} / T_{\infty}, 3 m_{\mathrm{e}} / m_{1}$ and $R / \sqrt{\varepsilon \lambda_{\infty} l_{\mathrm{e} \infty}}$ (as against $R / l_{\mathrm{e} \infty}$ for fully classical transport, as a result of changing the $z$-scale from (5a) to (19)), in addition to Braginskii's parallel transport coefficients $\alpha_{0}, \beta_{0}, \gamma_{0}$, and thermoelectric coefficients $\beta_{1}^{\prime \prime}, \gamma_{1}^{\prime}$. Also, equations (4') and (6) differ from the corresponding equations in the classical case because a constant factor $\varepsilon$ is replacing $1 / \Omega_{\mathrm{e}} \tau_{\mathrm{e}}$, which varied as $n / T_{\mathrm{e}}^{3 / 2}$. (A factor 2 was missing from the right-hand side of equation $\left(12^{\prime}\right)$ of [2].)

Numerical solutions were obtained by using full multigrid algorithm (FMG) and full aproximation storage algorithm (FAS) techniques. The choices as smoother, restriction opertor and prolongation operator were the Gauss-Seidel scheme, full weighting operator and bilinear interpolation, respectively. Results are presented for values of $R / \sqrt{ } \varepsilon \lambda_{\infty} l_{\mathrm{e} \infty}$ up to 90 , for which the scheme was efficient as regards convergence. Beyond this parameter range convergence becomes progressively slower, finally breaking down at high enough $R / \sqrt{ } \varepsilon \lambda_{\infty} l_{\mathrm{e} \infty}$.

\section{Discussion of results}

Figures 1 and 2 show the potential at the base of the outer region averaged over the probe, $\left\langle\phi_{0}\right\rangle \equiv \int_{0}^{R} \phi_{0} 2 r \mathrm{~d} r / R^{2}$. Finding $\phi_{0}>0$ at negative bias means that the potential overshoots its (zero) faraway value [13]. Positive $\phi_{0}$ values at $\phi_{\mathrm{P}}<0$ arise from electron cross-flow being inhibited by the magnetic field, which makes a parallel electron flux to persist over long distances, allowing ion-electron resistivity to keep it low. For non-emissive probes, a low 


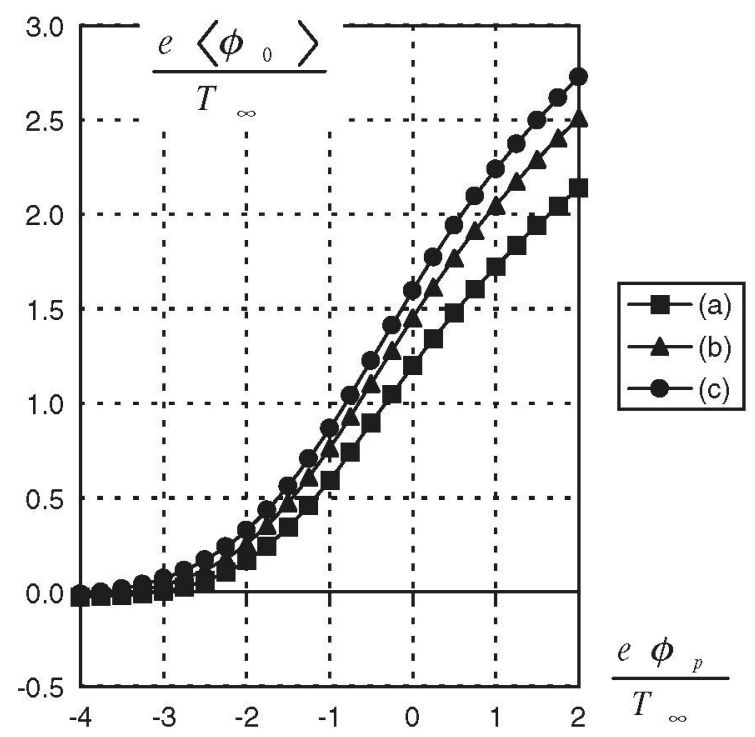

Figure 1. Potential at the base of the outer region averaged over the probe cross section, $\left\langle\phi_{0}\right\rangle \equiv \int_{0}^{R} 2 r \mathrm{~d} r \phi_{0} / R^{2}$. Thermoelectric coefficients $\gamma_{1}^{\prime}=4.664$ and $\beta_{1}^{\prime \prime}=0 ; m_{\mathrm{i}} / m_{\mathrm{e}}=$ $3672 ; R / \sqrt{ } \varepsilon \lambda_{\infty} l_{\mathrm{e} \infty}=30(a), 60(b), 90(c)$.

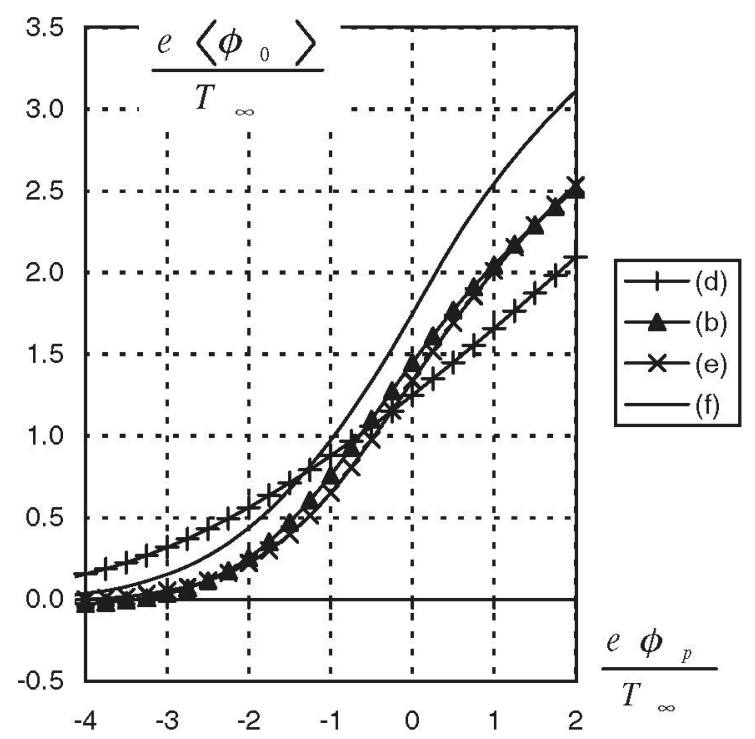

Figure 2. Average base potential for the conditions of case $(b)$ in figure 1, using an isothermal approximation with equations $\left(6^{\prime}\right)$ and $\left(21^{\prime}\right)$ ignored $(d)$; thermoelectric coefficient $\beta_{1}^{\prime \prime}=\frac{3}{2}(e)$; or fully classical transport as in section 2 with $R / l_{\mathrm{e} \infty}=150(f)$.

electron flux requires a low electron density; quasineutrality and the Boltzmann law for ions then result in positive $\phi_{0}$. Figures 1 and 2 show that $\phi$ remains nonmonotonic, validating our analysis, until probe bias catches up with $\phi_{0}$ at about $e \phi_{\mathrm{P}} / T_{\infty} \sim+2$.

The average base potential $\left\langle\phi_{0}\right\rangle$ does, indeed, increase with field $B$ and cross-flow inhibition in figure 1 , in agreement with the argument above. Figure 2 shows that reverting to 


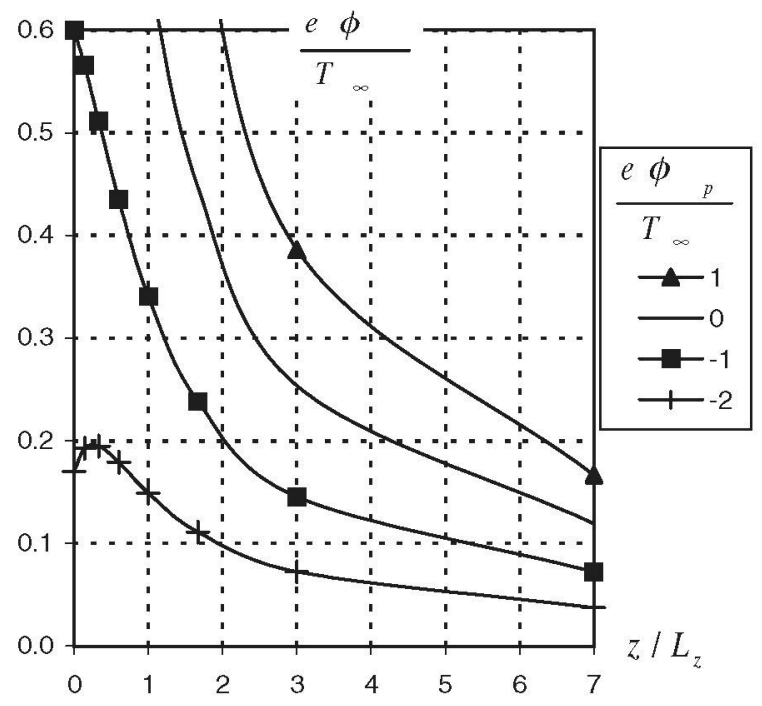

Figure 3. Potential profile along the probe axis in the outer region; $\gamma_{1}^{\prime}=4.664$ and $\beta_{1}^{\prime \prime}=0$; $m_{\mathrm{i}} / m_{\mathrm{e}}=3672 ; R / \sqrt{ } \varepsilon \lambda_{\infty} l_{\mathrm{e} \infty}=30$.

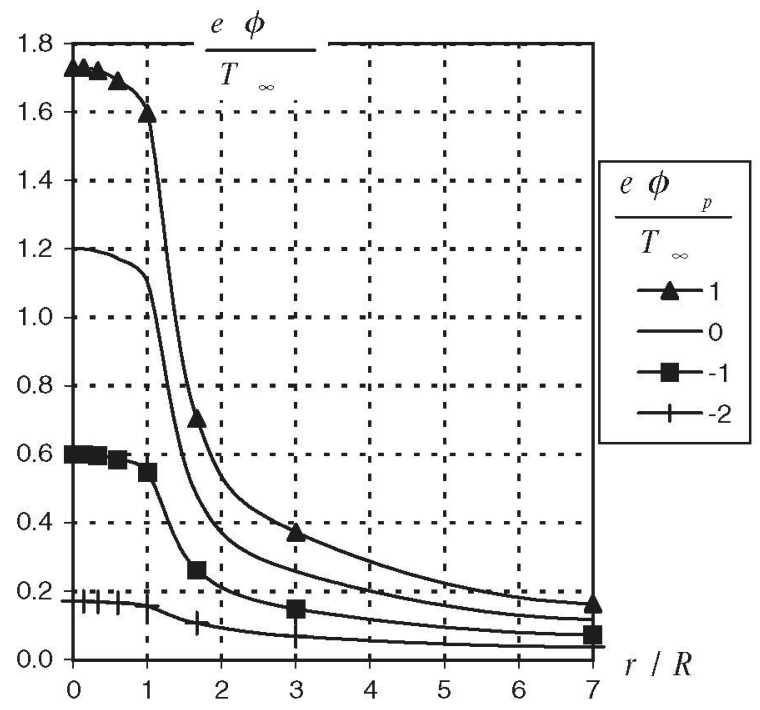

Figure 4. Radial potential profile at the base of outer region; conditions as in figure 3.

fully classical transport and its weaker cross-field diffusion, again increases $\left\langle\phi_{0}\right\rangle$. On the other hand, using the thermoelectric value $\beta_{1}^{\prime \prime}=\frac{3}{2}$ instead of $\beta_{1}^{\prime \prime}=0$ has hardly an effect. As regards an isothermal approximation that ignores equations $\left(6^{\prime}\right)$ and $\left(21^{\prime}\right)$, figure 2 shows $\left\langle\phi_{0}\right\rangle$ keeping much higher at very negative $\phi_{\mathrm{P}}$. Maximum $\tilde{\phi}(\tilde{z})$ values are not very different from nonisothermal cases, however. Note that setting $\partial \tilde{T} /\left.\partial \tilde{z}\right|_{0}=0$ in $\left(20^{\prime}\right)$ makes $\partial \tilde{\phi} /\left.\partial \tilde{z}\right|_{0}$ negative, whereas considering both $\left(20^{\prime}\right)$ and $\left(21^{\prime}\right)$ makes $\partial \tilde{\phi} /\left.\partial \tilde{z}\right|_{0}$ (as well as $\partial \tilde{T} /\left.\partial \tilde{z}\right|_{0}$ ), negative and positive at the highest and lowest bias, respectively. A positive $\partial \tilde{\phi} /\left.\partial \tilde{z}\right|_{0}$ makes $\operatorname{Max} \tilde{\phi}(\tilde{z})>\tilde{\phi}_{0}$ : thermal effects may set the potential overshoot off the base of the outer region as shown in 


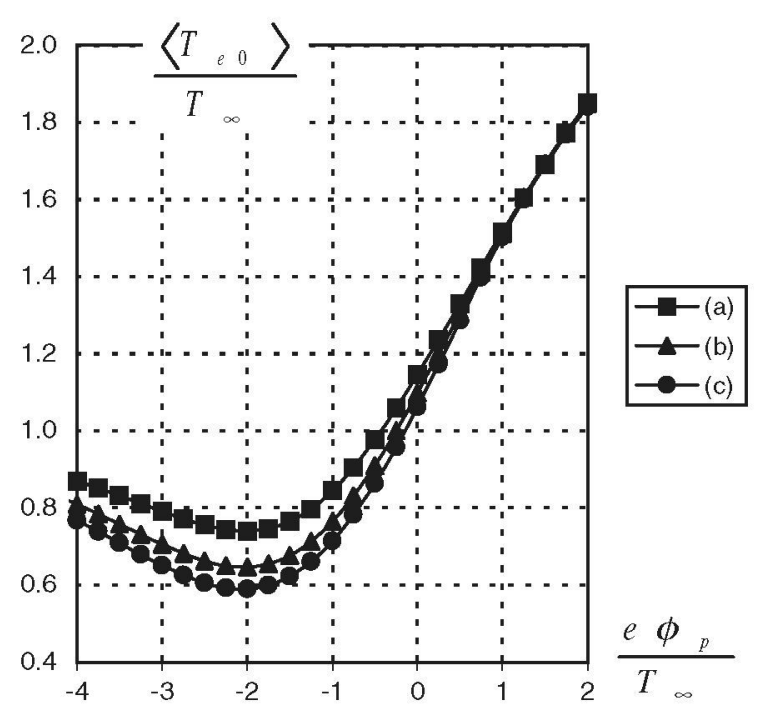

Figure 5. Average electron temperature at the base of outer region, $\left\langle T_{\mathrm{e} 0}\right\rangle \equiv \int_{0}^{R} 2 r \mathrm{~d} r T_{\mathrm{e} 0} / R^{2}$, for the conditions of figure 1 .

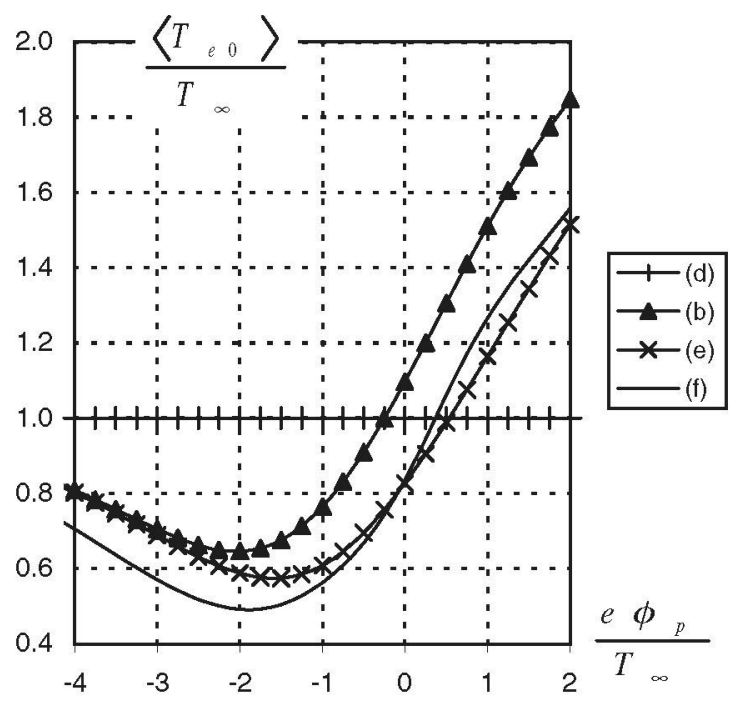

Figure 6. Average of base electron temperature for the conditions of figure 2.

figure 3; this occurs at $e \phi_{\mathrm{P}} / T_{\infty}$ as high as -2 . Figure 4 presents the radial potential profile at the base of the outer region.

Figures 5 and 6 show the average base temperature $\left\langle T_{\mathrm{e} 0}\right\rangle$. There is heating and cooling at the highest and lowest bias, roughly corresponding to the negative and positive $\partial \tilde{T} /\left.\partial \tilde{z}\right|_{0}$ values noted above. The behaviour of electron temperature near the probe is thus basically determined by boundary conditions $\left(20^{\prime}\right)$ and $\left(21^{\prime}\right)$. Clearly, the temperature minimum is a result of cooling necessarily vanishing with the electron current as $\phi_{\mathrm{P}}$ becomes negative enough.

Note that thermoelectric terms $\left(\beta_{1}^{\prime \prime}=\frac{3}{2}\right.$ ) do have a sensible effect on cooling in figure 6 . Also, cooling does increase with cross-flow inhibition (either greater $B$ in figure 5, or moving 


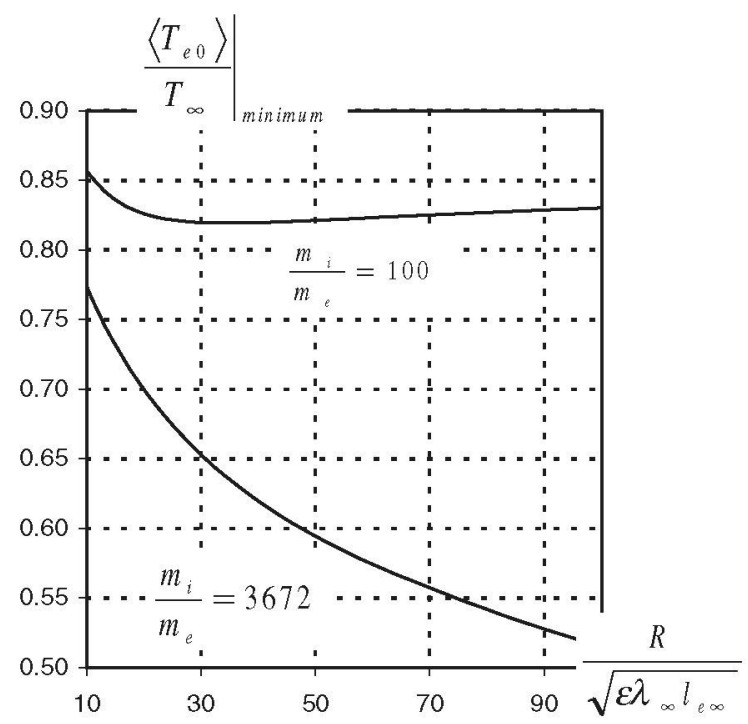

Figure 7. Minimum of electron temperature versus probe bias as a function of normalized radius; $\gamma_{1}^{\prime}=4.664, \beta_{1}^{\prime \prime}=\frac{3}{2}$.

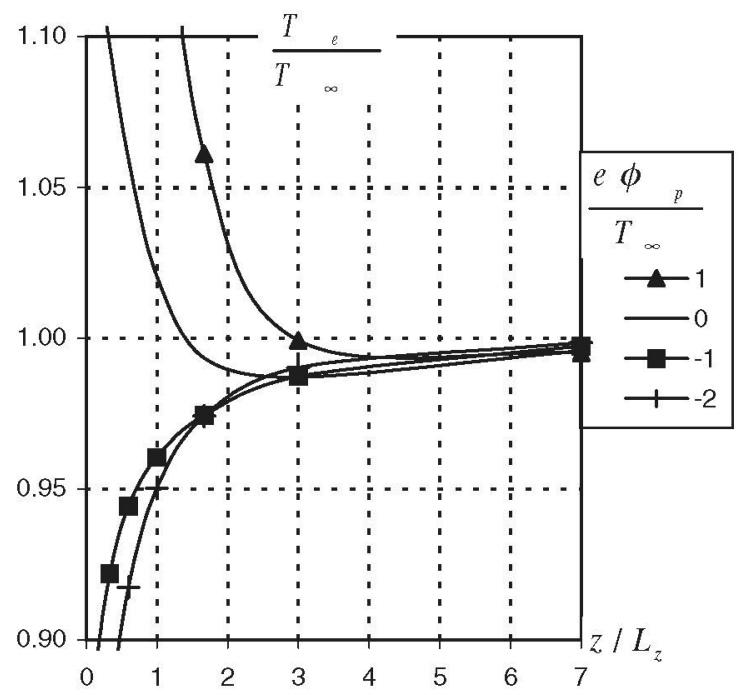

Figure 8. Electron temperature profile along probe axis in the outer region; conditions as in figure 3.

to fully classical transport in figure 6). Ultimately, however, the $z$-scale would become so large that the $Q_{\mathrm{i}}$ term for ion-electron heat exchange in equation (6) would be able to keep $T_{\mathrm{e}}$ close to the ion temperature $T_{\infty}$. The $T_{\mathrm{e}}$ versus $\phi_{\mathrm{P}}$ minimum will thus have an extremum at certain large $R / \sqrt{\varepsilon \lambda_{\infty} l_{\mathrm{e} \infty}}$ value. Both that value and the extremum itself are greater the greater is the mass ratio $m_{\mathrm{i}} / m_{\mathrm{e}}$ (figure 7 ).

Temperature behaviour far from the probe, on the other hand, is weakly dependent on probe bias. Figure 8 shows $\tilde{T}(\tilde{r}=0, \tilde{z})$; there is faraway cooling for all $\phi_{\mathrm{P}}$. Heat conduction is here determinant. In fact, if $\bar{q}_{\mathrm{e}}$ were ignored in equation (6), heating would necessarily 


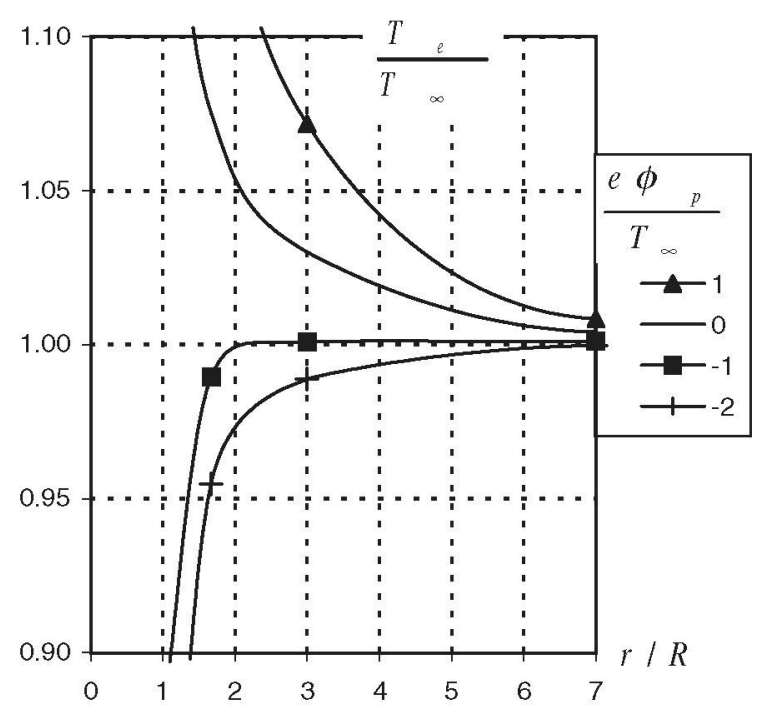

Figure 9. Radial profile of electron temperature at the base of outer region; conditions as in figure 3 .

occur. Using (4), equation (6) on the $r$-axis would yield

$$
\frac{5}{2}\left(T_{\infty}-T_{\mathrm{e}}\right)+e \phi=\int_{z}^{\infty} \mathrm{d} z^{\prime} \frac{Q_{\mathrm{i}}}{-n v_{\mathrm{e} z}} ;
$$

with $\phi$ positive, equation (22) implies $T_{\mathrm{e}}>T_{\infty}$. Actually, one can use (4) to verify that, far from the probe, terms other than $\nabla \cdot \bar{q}_{\mathrm{e}}$ on the left-hand side of (6) are of higher order compared with $\nabla \cdot \bar{q}_{\mathrm{e}}$. Figure 9 shows $\tilde{T}(\tilde{r}, \tilde{z}=0)$; here there is faraway heating for all $\phi_{\mathrm{P}}$.

Ignoring the variations of $\phi_{0}$ and $T_{\mathrm{e} 0}$ across the probe, equation (20) can be written as

$$
\ln I_{\mathrm{e}} \approx \frac{1}{2} \ln \frac{T_{\mathrm{e} 0}}{T_{\infty}}-\frac{e\left(\phi_{0}-\phi_{\mathrm{P}}\right)}{T_{\mathrm{e} 0}}-\frac{e \phi_{0}}{T_{\infty}}+\text { const. }
$$

where the electron current $I_{\mathrm{e}}$ decreases both with increasing $\phi_{0}$ and decreasing $T_{\mathrm{e} 0}$. Our results show $I_{\mathrm{e}}$ indeed decreasing with reduced cross-flow, in agreement with (23), as either $B$ is increased (figure 10) or classical transport is considered (curve (f) in figure 11). At $\phi_{\mathrm{P}}$ negative enough, with $\phi_{0} \approx 0, T_{\mathrm{e} 0} \approx T_{\infty}$, the slope $\mathrm{d}\left(\ln I_{\mathrm{e}}\right) / \mathrm{d}\left(e \phi_{\mathrm{P}}\right) \approx 1 / T_{\infty}$ in $(23)$ is just the inverse of the unperturbed electron temperature, as in unmagnetized plasmas. At higher bias one might expect that cooling would result in the slope increasing with decreasing $T_{\mathrm{e} 0}$ but figures 10 and 11 show otherwise. This fact is now shown to arise from $T_{\mathrm{e} 0}$ and $\phi_{0}$ being $\phi_{\mathrm{P}}$-dependent [2].

Taking the derivative of equation (23) with respect to $\phi_{\mathrm{P}}$ yields

$$
\frac{\mathrm{d} \ln I_{\mathrm{e}}}{\mathrm{d} e \phi_{\mathrm{P}}}=\frac{1}{T_{\mathrm{e} 0}}\left[1-\left(1+\frac{T_{\mathrm{e} 0}}{T_{\infty}}\right) \frac{\mathrm{d} \phi_{0}}{\mathrm{~d} \phi_{\mathrm{P}}}+\left(\frac{1}{2}+e \frac{\phi_{0}-\phi_{\mathrm{P}}}{T_{\mathrm{e} 0}}\right) \frac{\mathrm{d} T_{\mathrm{e} 0}}{\mathrm{~d} e \phi_{\mathrm{P}}}\right] .
$$

In the isothermal model the slope is $\left(1-2 \mathrm{~d} \phi_{0} / \mathrm{d} \phi_{\mathrm{P}}\right) / T_{\infty}$; with $\mathrm{d} \phi_{0} / \mathrm{d} \phi_{\mathrm{P}}$ positive and increasing with $\phi_{\mathrm{P}}$, the slope is less than $1 / T_{\infty}$ and decreasing with $\phi_{\mathrm{P}}$, resulting in a graph that is concavedownwards, as seen in curve (d) of figure 11. On the other hand, in non-isothermal cases under strong cross-flow inhibition, the graph becomes concave-upwards as seen in curves (b) $\left(\beta_{1}^{\prime \prime}=0\right)$ and (e) $\left(\beta_{1}^{\prime \prime}=\frac{3}{2}\right)$. This fact relates to the temperature minimum. The last term in (24) is then large and changes sign at the minimum, making the graph tilt upwards past it, a feature found 


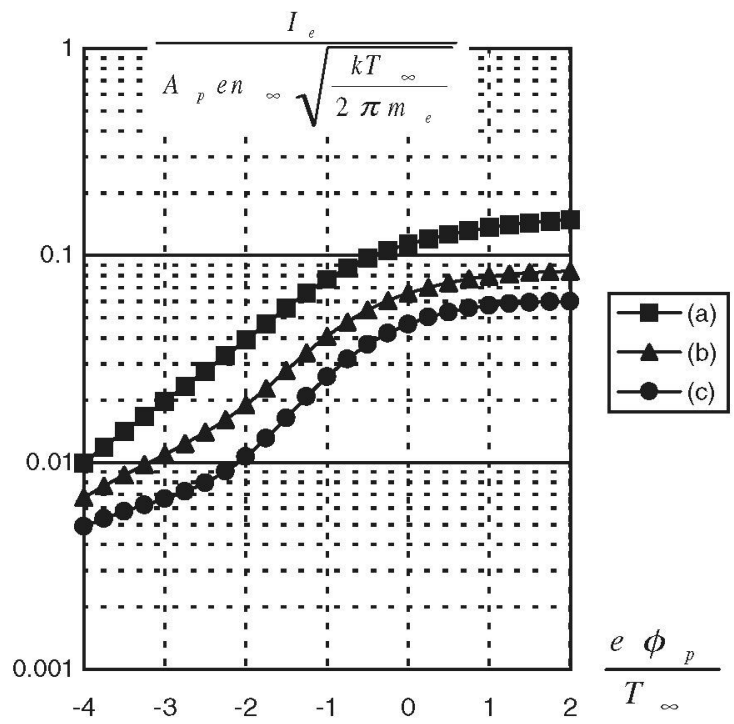

Figure 10. Normalized electron current for the conditions of figure $1\left(A_{\mathrm{P}}=2 \pi R^{2}\right)$.

in some experiments and displayed in unmagnetized plasmas with two electron temperatures $[19,35]$.

To compare our electron 'saturation current' with Bohm's estimate [12] as modified by Stangeby [33] one would write

$$
n v_{\mathrm{ez}}=-\frac{2 D_{\mathrm{e} z}\left(n_{\infty}-n_{0}\right)}{l_{z}}, \quad n v_{\mathrm{e} r}=-\frac{2 D_{\mathrm{e} \perp}\left(n_{\infty}-n_{0}\right)}{2 R},
$$

with $n v_{\mathrm{e} z}=-n_{0} c_{\mathrm{e}} / \sqrt{ } 2 \pi$ and $n v_{\mathrm{er}} / n v_{\mathrm{e} z}=\pi R^{2} / 2 \pi R l_{z}$; here electron fluxes are supposed to represent average values. Using $D_{\mathrm{e} z}=c_{\mathrm{e}}^{2} \tau_{\mathrm{e}} / \alpha_{0}$ and $D_{\mathrm{e} \perp}=\varepsilon c_{\mathrm{e}}^{2} / \Omega_{\mathrm{e}}$ one finds

$$
\frac{\sqrt{2 \pi}}{n_{\infty} c_{\mathrm{e}}} \times n v_{\mathrm{e} z}=\frac{r_{\mathrm{St}}}{1+r_{\mathrm{St}}}, \quad r_{\mathrm{St}} \equiv 2 \sqrt{\frac{2 \pi}{\alpha_{0}}} \times \frac{\sqrt{\varepsilon \lambda_{\infty} l_{\mathrm{e} \infty}}}{R} .
$$

For the case of figure $11\left(R / \sqrt{\varepsilon \lambda_{\infty} l_{\mathrm{e} \infty}}=60\right)$ we get $r_{\mathrm{St}} \approx 0.117$ and a normalized current, $r_{\mathrm{St}} /\left(1+r_{\mathrm{St}}\right) \approx 0.105$, close to the value 0.112 at $e \phi_{\mathrm{P}} / T_{\infty}=2$ in our results without thermal effects (curve (d)). Also, currents and hill-potentials for the entire retarding range that result from the average analysis of [33] can be shown to be close to corresponding values in curves (d) of figures 11 and 2 : taking $r_{\mathrm{St}} \approx 0.117$, Stangeby's normalized currents at $e \phi_{\mathrm{P}} / T_{\infty}=-2(0)$ would be $0.048(0.083)$, compared with d-curve values 0.044 (0.082) in figure 11; hill potentials at $e \phi_{\mathrm{P}} / T_{\infty}=-2(2)$ would be $e \phi_{0} / T_{\infty}=0.522$ (2.135), compared with d-curve values 0.563 (2.094) in figure 2 .

Although saturation current and radial averaging, say, are ill defined, the standard average analysis in [33] does approximate our no-thermal-effects results. We now note that thermal effects do affect collection substantially (and are greater when taking the classical value $\beta_{1}^{\prime \prime}=\frac{3}{2}$ instead of $\beta_{1}^{\prime \prime}=0$ ): the current in curve (e) of figure 11 is smaller than current in curve (d) by as much as half-an-order of magnitude; the saturation current in curve (e) is about half the current in curve (d). As a consequence, thermal effects might explain why standard analyses, which have always ignored such effects, usually predict values of current that are sensibly greater than measured values [33]. 


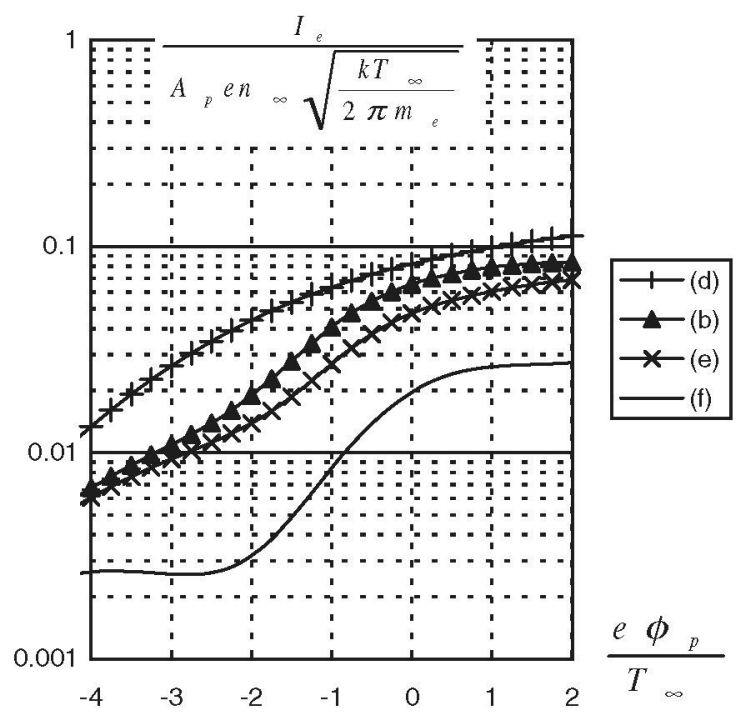

Figure 11. Normalized electron current for the conditions of figure 2.

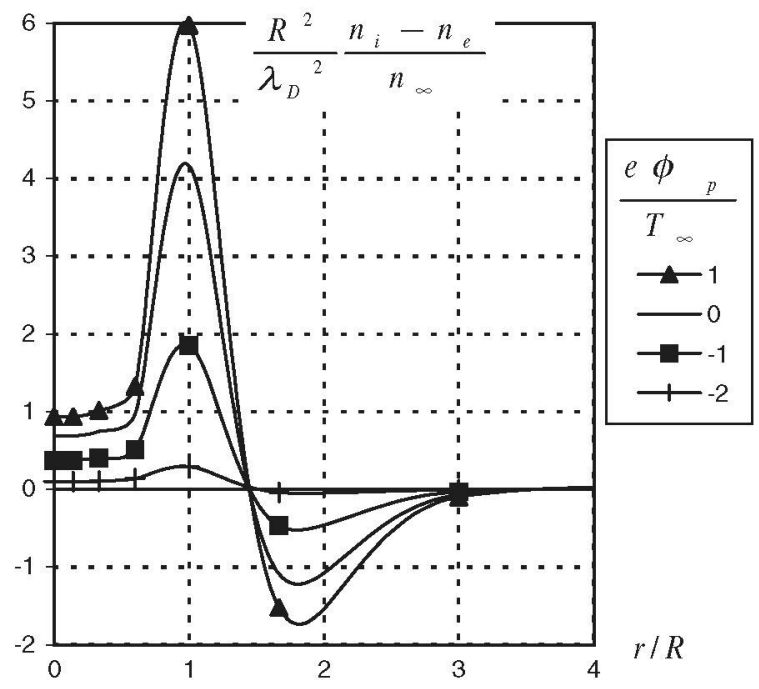

Figure 12. Radial profile of space charge at the base of outer region; conditions as in figure 3 .

Finally, results for $\tilde{\phi}(\tilde{r}, \tilde{z})$ can be used in Poisson's equation to determine the spatial structure of the resulting weak space charge. Using $R^{2} / L_{z}^{2}=\alpha_{0} \varepsilon l_{\mathrm{e} \infty} / \lambda_{\infty} \ll 1$ one finds

$$
-\frac{R^{2}}{\lambda_{\mathrm{D}}^{2}} \frac{n_{\mathrm{i}}-n_{\mathrm{e}}}{n_{\infty}}=\frac{1}{\tilde{r}} \frac{\partial}{\partial \tilde{r}} \tilde{r} \frac{\partial \tilde{\phi}}{\partial \tilde{r}}+\frac{\alpha_{0} \varepsilon l_{\mathrm{e} \infty}}{\lambda_{\infty}} \frac{\partial^{2} \tilde{\phi}}{\partial \tilde{z}^{2}} \approx \frac{1}{\tilde{r}} \frac{\partial}{\partial \tilde{r}} \tilde{r} \frac{\partial \tilde{\phi}}{\partial \tilde{r}},
$$

where $\lambda_{\mathrm{D}}(\ll R)$ is the Debye length. Note that because of the large discrepancy in $z$ and $r$ scales, radial profiles determine charge separation everywhere. Figure 12 shows the space charge at $\tilde{z}=0$ : the double layer, with ion (electron) excess at lower (greater) radius, is directly related to radial potential profiles in figure 4 , which are downwards (upwards) concave at lower (greater) radius; note that consideration of the mostly upwards concave profiles along 


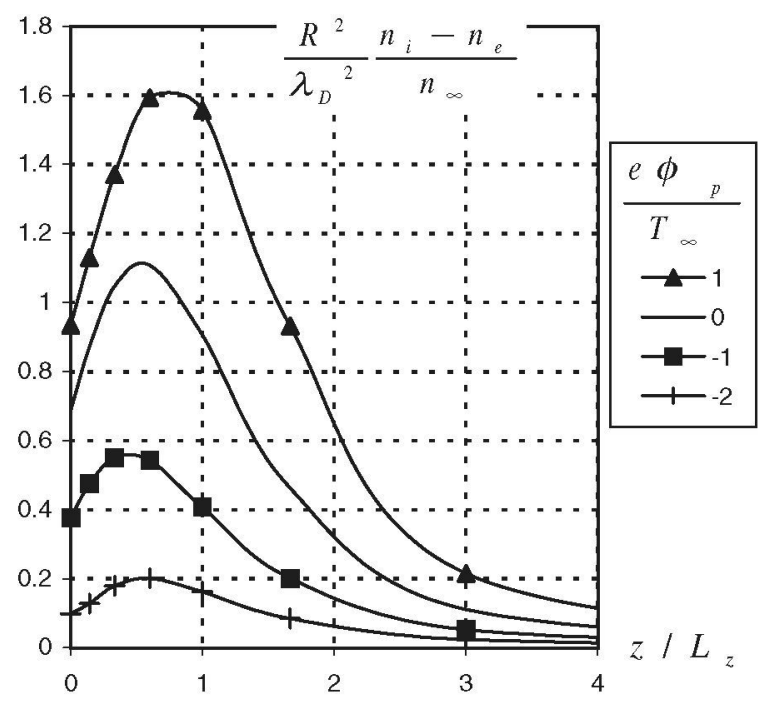

Figure 13. Space charge profile along the probe axis in outer region; conditions as in figure 3.

$z$ in figure 3 would have wrongly suggested electron excess at $\tilde{r}=0$ [16]. Figure 13 for the space charge at $\tilde{r}=0$ (where $\tilde{r}^{-1}(\partial / \partial \tilde{r}) \tilde{r} \partial \tilde{\phi} / \partial \tilde{r}$ does not vanish) shows the double layer structure peaking before decreasing along the magnetic field. Independently, note that contrary to the case of equation (25), all terms in equation (4') and (the left-hand side of) equation (6') are comparable. That means that describing transport in our magnetized plasma as a onedimensional problem because cross-field diffusion is much weaker than parallel diffusion is an often used approximation not more valid than in the case of no magnetic field.

\section{Model validity}

With $v_{i \theta}$ ignored in (17) and (18), ansatzen (i), (iii) and (iv) just require $\varepsilon \lambda l_{\mathrm{e}} / R^{2}$ and $\sqrt{m_{\mathrm{e}} / m_{\mathrm{i}}} \times R^{2} / l_{\mathrm{i}}^{2}$ to be small. These conditions determine an allowed range of probe radius,

$$
\sqrt{\varepsilon \lambda l_{\mathrm{e}}} \ll R \ll l_{\mathrm{i}} \sqrt[4]{\frac{m_{\mathrm{i}}}{m_{\mathrm{e}}}} .
$$

Actually length disparities in (26) need be just moderately large, model validity involving the square of these lengths. The implied inequality in (26),

$$
\frac{\varepsilon \lambda}{l_{\mathrm{i}}} \ll \frac{m_{\mathrm{i}}}{m_{\mathrm{e}}},
$$

is well satisfied. Note, however, that $R / \sqrt{\varepsilon \lambda / l_{\mathrm{e}}}$ will need be quite large for the $\ln I_{\mathrm{e}}-\phi_{\mathrm{P}}$ graph to exhibit upwards concavity (figure 10).

Turning to $v_{\mathrm{i} \theta}$, the Boltzmann law for ions will break down in case that velocity is large enough. Quasineutral density and azimuthal-field fluctuations sustaining anomalous transport might drive the average ion $\theta$-momentum equation the way they drive the corresponding electron equation, scaling up $v_{\mathrm{i} \theta}$ along with $v_{\mathrm{e} r}$. Since $v_{\mathrm{ir}} / v_{\mathrm{e} r}$ was again small in section 3, viscosity would still be crucial for ion $\theta$-momentum balance. Similar to the result $F_{\mathrm{i} \theta}^{\mathrm{v}} \approx R_{\mathrm{e} \theta} \approx$ $-e B n v_{\mathrm{e} r}$ arising from equations (1b) and (8b), one would then obtain

$$
F_{\mathrm{i} \theta}^{\mathrm{v}}\left(v_{\mathrm{i} \theta}\right) \approx \varepsilon\left[\frac{\partial p_{\mathrm{e}}}{\partial r}-e n \frac{\partial \phi}{\partial r}-\beta_{1}^{\prime \prime} n \frac{\partial T_{\mathrm{e}}}{\partial r}\right] \approx-e B n v_{\mathrm{e} r}
$$


and using (9b) arrive at

$$
\begin{aligned}
& \frac{v_{1 \theta}}{c_{1}} \sim \frac{\varepsilon \lambda}{l_{1}} \times \frac{R}{l_{1}}, \\
& \frac{v_{1 \theta}}{v_{\mathrm{e} \theta}} \sim \frac{\varepsilon \lambda}{l_{1}} \times \frac{R^{2}}{l_{1}^{2}} .
\end{aligned}
$$

Allowing for all dominant $v_{1 \theta}$-terms, equation (14) now reads

$$
m_{1} n \frac{v_{1 \theta}^{2}}{r} \approx-T_{\infty} \frac{\partial n}{\partial r}-e n \frac{\partial \phi}{\partial r}+F_{1 r}^{\mathrm{v}}\left(v_{1 \theta}\right)+e B n v_{1 \theta} .
$$

Compared with the first two terms on the right-hand side, inertia, viscous, and magnetic terms are of order $v_{1 \theta}^{2} / c_{1}^{2},\left(v_{1 \theta} / c_{1}\right) \times l_{1} / R$, and $\left(v_{1 \theta} / c_{1}\right) \times R / l_{1}$, respectively. For these three terms, and for $v_{1 \theta} / v_{\mathrm{e} \theta}$ in $(29 b)$, to be negligible, both $\varepsilon \lambda / l_{1}$ and $\left(R / l_{1}\right)^{2} \times \varepsilon \lambda / l_{1}$ need be small, requiring

$$
\begin{aligned}
& \frac{\varepsilon \lambda}{l_{1}} \approx 0.088 \frac{B(\mathrm{~T})}{n\left(10^{20} \mathrm{~m}^{-3}\right)} \frac{\left[T_{\mathrm{e}}(\mathrm{eV})\right]^{3 / 2}}{A_{1}^{1 / 2}} \ll 1, \\
& \sqrt{\varepsilon \lambda l_{\mathrm{e}}}<R<l_{1} \sqrt{\frac{l_{1}}{\varepsilon \lambda}} \approx 0.344 \frac{\left[n\left(10^{20} \mathrm{~m}^{-3}\right)\right]^{1 / 2}}{[B(\mathrm{~T})]^{3 / 2}} \frac{A_{1}^{3 / 4}}{\left[T_{\mathrm{e}}(\mathrm{eV})\right]^{1 / 4}} \mathrm{~mm},
\end{aligned}
$$

where $A_{1}$ is the atomic number; we set Coulomb logarithm $=10$ and $\varepsilon=\frac{1}{16}$, and used the previous condition $\varepsilon \lambda l_{\mathrm{e}} / R^{2} \ll 1$ for the lower end of the range in $\left(26^{\prime}\right)$. Since model validity again involves the square of lengths in $\left(26^{\prime}\right)$, we wrote these conditions in terms of simple inequality signs. Also, since $\varepsilon \lambda / l_{\mathrm{e}}$ is supposed to be large, the upper end in $\left(26^{\prime}\right)$ is more stringent than in (26). The new length range implies the inequality

$$
\left(\varepsilon \lambda / l_{1}\right)^{2} \ll \sqrt{\frac{m_{1}}{m_{\mathrm{e}}}} .
$$

Conditions (30) and $\left(26^{\prime}\right)$ are easier to meet the higher the ion mass. Consider argon ions $\left(A_{1} \approx 40\right)$ and take $\varepsilon=\frac{1}{16}, T_{\infty}=5 \mathrm{eV}, n_{\infty}=5 \times 10^{18} \mathrm{~m}^{-3}, B=0.05 \mathrm{~T}$ [9]. We find

$$
\lambda_{\infty} \approx 72 \mathrm{~mm}, \quad l_{1 \infty} \approx 28.8 \mathrm{~mm}, \quad \varepsilon \lambda_{\infty} / l_{1 \infty} \approx 0.156,
$$

and, using $\left(26^{\prime}\right)$,

$$
0.7 \mathrm{~mm}<R<72.9 \mathrm{~mm} \text {. }
$$

A typical valid radius would then be $20 \mathrm{~mm}$. As density and magnetic field are increased valid probes would be smaller; for $n_{\infty}=10^{20} \mathrm{~m}^{-3}$ and $B=1 \mathrm{~T}[10]$, we have $\lambda_{\infty} \approx 3.6 \mathrm{~mm}$, $l_{1 \infty} \approx 1.44 \mathrm{~mm}, \varepsilon \lambda_{\infty} / l_{1 \infty} \approx 0.156$,

$$
0.035 \mathrm{~mm}<R<3.65 \mathrm{~mm} \text {. }
$$

Consider now deuterium ions, with values $n_{\infty}=10^{20} \mathrm{~m}^{-3}, B=0.5 \mathrm{~T}$. Valid temperatures should now be smaller to keep $\varepsilon \lambda / l_{1}$ small, and keep the upper-end $R$-range from unreasonable shrinking in $\left(27^{\prime}\right)$. For $T_{\infty}=1 \mathrm{eV}$ we find

$$
0.0046 \mathrm{~mm}<R<1.65 \mathrm{~mm} \text {. }
$$

We note that thermal (and current $I_{\mathrm{e}}$ ) effects are more pronounced for higher ion mass (figure 7 ) and lower cross-field diffusion (lower $\varepsilon$, with a minimum, classical value, $l_{\mathrm{e}} / \lambda$ ).

Our model will fully break down with Boltzmann's law in case $\varepsilon \lambda / l_{1}$ or $\left(R / l_{1}\right)^{2} \times \varepsilon \lambda / l_{1}$ is large. On the other hand, if they are just of order unity, results, though quantitatively different, will retain the basic qualitative character of figures 1-13. Actually, when a linear analysis applies (at the lower values of $R / \sqrt{\varepsilon \lambda_{\infty} l_{\mathrm{e} \infty}}$, or for bias to left and away from the 
$T_{\mathrm{e} 0}$ minimum in figure 5 at all $R / \sqrt{\varepsilon \lambda_{\infty} l_{\mathrm{e} \infty}}$ ), results on both $T_{\mathrm{e}}$ and $I_{\mathrm{e}}$ in section 4 are independent of equation (15b). If this Boltzmann law is not used, equations (4), (6), (20), and (21) involve all three quantities, $T_{\mathrm{e}}, \phi$, and $n$; when linearized, however, they involve just $\tilde{T}-1$ and $\tilde{\phi}+1-n / n_{\infty}$. If the linear form of $(15 b), n / n_{\infty}=1-\tilde{\phi}$, were now used, one would reproduce a direct linear analysis of equations $\left(4^{\prime}\right),\left(6^{\prime}\right),\left(20^{\prime}\right)$ and $\left(21^{\prime}\right)$. Thus, in a linear regime, one may solve for current and temperature (and the combination $e \phi-T_{\infty} \ln n$ ) without recourse to the ion Boltzmann law, results being the same as if equation (15b) were used.

At greater $R / \sqrt{\varepsilon \lambda_{\infty} l_{\mathrm{e} \infty}}$, the linear analysis cannot describe, even if roughly, behaviour around the temperature minimum. We can estimate, however, the effects of Boltzmann's law breakdown, if weak. We may drop the left-hand side of $\left(14^{\prime}\right)$, which would be a correction of higher order, and use (28) to rewrite equation (14') as

$$
\begin{aligned}
& \frac{\partial \ln n}{\partial r}+\frac{\partial \tilde{\phi}}{\partial r}=\varepsilon^{\mathrm{v}}\left[\tilde{T} \frac{\partial \ln n}{\partial r}-\frac{\partial \tilde{\phi}}{\partial r}+\left(1-\beta_{1}^{\prime \prime}\right) \frac{\partial \tilde{T}}{\partial r}\right], \\
& \varepsilon^{\mathrm{v}} \equiv \varepsilon \times \frac{F_{1 F}^{\mathrm{v}}\left(v_{1 \theta}\right)+n T_{\infty} v_{1 \theta} / \Omega_{1} l_{1}^{2}}{F_{1 \theta}^{\mathrm{v}}\left(v_{1 \theta}\right)},
\end{aligned}
$$

with the right-hand side of (31) as a small correction. The first and second $\varepsilon^{\mathrm{v}}$-terms are positive and negative, respectively (with $-v_{\mathrm{e} r}$, and thus $F_{1 \theta}^{\mathrm{v}}\left(v_{1 \theta}\right)$, positive in (28), $v_{1 \theta}$ may be proved negative if constant in sign in the range $0<r<\infty$; then $F_{1 r}^{\mathrm{v}}\left(v_{1 \theta}\right)$ comes out positive). Hence, $\varepsilon^{\mathrm{v}}$ will change from positive to negative as $\left(R / l_{1}\right)^{2}$ is increased. We solve (31) iteratively, set $\tilde{T} \approx 1$ in the first term of the bracket, use some average $\varepsilon_{\mathrm{av}}^{\mathrm{v}}$, and take $\beta_{1}^{\prime \prime}=0$, to write

$$
\ln \frac{n}{n_{\infty}} \approx-\left[1+2 \varepsilon_{\mathrm{av}}^{\mathrm{v}}\right] \frac{\mathrm{e} \phi}{T_{\infty}}-\varepsilon_{\mathrm{av}}^{\mathrm{v}}\left(1-\frac{T_{\mathrm{e}}}{T_{\infty}}\right) .
$$

Since $\phi$ and $1-T_{\mathrm{e}} / T_{\infty}$ are positive, both corrections to Boltzmann's law above have sign opposite $\varepsilon^{\mathrm{v}}$ and should have a similar effect. The first correction is equivalent to a change in ion temperature from $T_{\infty}$ to $T_{\infty} /\left(1+2 \varepsilon_{\mathrm{av}}^{\mathrm{v}}\right)$; the overall effect should just be equivalent to a decrease (increase) in ion temperature at the lower (higher) $R^{2} / l_{1}^{2}$ values. We then note that in the fully collisional case, a decrease in $T_{1 \infty} / T_{\mathrm{e} \infty}$ reduces the current beyond $\phi_{\mathrm{P}}=0$, and both reduces $\phi_{0}$ and increases $T_{\mathrm{e} 0}$ beyond $\phi_{\mathrm{P}}$ at the temperature minimum (see figures 4-6 of [2]).

\section{Conclusions}

We have allowed for thermal effects and anomalous (Bohm) cross-field transport in consistently studying the electron-retarding range of the $\mathrm{C}-\mathrm{V}$ characteristic of a probe in a strongly magnetized, collisional plasma. Length ordering is, broadly, $l_{\mathrm{e}} \ll \varepsilon \lambda<R \sim l_{1}<\lambda$, where $R$ is the probe radius, $l_{\mathrm{e}}, l_{1}$, and $\lambda$ are electron and ion thermal gyroradii and electron mean free path, and $\varepsilon$ is the factor in Bohm's diffusion coefficient (set at $\frac{1}{16}$ by Bohm). In the parametric domain of validity of our analysis there is a large outer region where ion density follows the Boltzmann law, and ion viscosity is determinant in allowing non-ambipolar quasineutral flow along with two separate continuity equations. The domain of validity is smaller than it was for classical cross-field transport, and is smaller the smaller is the ion mass.

The spatial structure of electron temperature $T_{\mathrm{e}}$ and potential $\phi$ (and density $n$ ) is complex. Potential $\phi$ overshoots its faraway value, extending the electron-retarding range to probe bias $\phi_{\mathrm{P}} \sim 2 T_{\mathrm{e} \infty} / e$; the overshoot lies either at the base or inside the outer region. Radial $\phi$ profiles determine a weak space-charge double layer, with ion density excess around the probe axis, parallel to the magnetic field. At the base of the outer region there is, roughly, heating and cooling at positive and negative bias, with a $T_{\mathrm{e}}$ minimum around $\phi_{\mathrm{P}} \sim-2 T_{\mathrm{e} \infty} / e$; far from 
the probe heat conduction results in cooling and heating at and radially away from the axis, whatever the bias

Reduction of electron current $I_{\mathrm{e}}$ by the magnetic field relates to the potential overshoot, which would just make the $\ln I_{\mathrm{e}}$ versus $\phi_{\mathrm{P}}$ graph downwards-concave if thermal effects were 1gnored Thermal effects further reduce the current, they may also t1lt the slope upwards past the temperature minimum, with the graph becoming upwards-concave there, a feature found in some experiments and displayed in unmagnetized plasmas with two electron temperatures Potential overshoot and current reduction are more pronounced the greater crossflow inhibition Cooling first increases, then decreases with increasing cross-flow inhibition, the $T_{\mathrm{e}}$-minımum has an extremum that is greater the greater is the 1on mass Valid probe sizes decrease as $10 n$ mass decreases or density and magnetic field increase

The greater current reduction found here when thermal effects are considered may expla1n why standard analyses, which have always 1gnored such effects, usually predict values of current that are sensibly greater than measured values [33] Use of our model in probe 1nterpretation will require a choice of Bohm's parameter $\varepsilon$ (to determine a ratio $R / \sqrt{ } \varepsilon \lambda_{\infty} l_{\mathrm{e} \infty}$ ), $1 \mathrm{e}$ a definite choice of cross-field diffusivity, the transport structure of our model being otherwise classical, cross-field mobility (and thermoelectric coefficients) are also determined once $\varepsilon$ is chosen In practice, unrelated cross-field diffusivity and mobility may need, be chosen In any case, independently of the detalled transport model, thermal effects such as found here will sensibly affect the values of current to the probe

\section{Acknowledgment}

Th1s work was supported by DGESIC of Spa1n under Grant No PB97-0574-C04-1

\section{References}

[1] Carlson A and Bergmann A $1999 \mathrm{~J}$ Nucl Mater 266-269 1020

[2] Charro M and Sanmartın J R 2000 Phys Plasmas 72622

[3] Laframboise J G and Sonmor L J 1993 J Geophys Res 98337

[4] Sanmartın J R and Estes R D 1999 Phys Plasmas 6395

Sanmartın J R and Estes R D 2001 Phys Plasmas 84234

[5] Estes R D and Sanmartın J R 2000 Phys Plasmas 74320

[6] Winnıngham J D et al 1998 Geophys Res Lett 25429

[7] Cooke D L and Katz I 1998 Geophys Res Lett 25753

[8] Samir U Israelevich P Wright K H and Stone N H 2001 J Geophys Res 10612963

[9] Gilmore M Peebles W A and Nguyen X V 2000 Plasma Phys Control Fuston 42 L1

[10] Carlson A 2001 Phys Plasmas 84732

[11] Gunn J P 1997 Phys Plasmas 44435

[12] Bohm D 1949 Characteristics of Electrcal Discharges in Magnetıc Fields ed A Guthrie and R K Wakerlıng (New York McGraw Hill)

[13] Sanmartin J R 1970 Phys Fluds 13103

[14] Nigoyı K K and Cohen I M 1973 Phys Flutds 1669

Kawaguch1 M and Tanaka H 1981 Phys Flutds $\mathbf{2 4} 2378$

[15] Pitts R A and Stangeby P C 1990 Plasma Phys Control Fuston 321237

[16] Cohen S A 1978 J Nucl Mater 76-77 68

[17] Stangeby P C 1982 J Phys D 151007

[18] Braginsk11 S I 1965 Revews of Plasma Physics vol 1 ed M A Leontovich (New York Consultants Bureau)

[19] Guenther K Herrmann A Laux M Pech P and Reıner H D $1990 \mathrm{~J}$ Nucl Mater 176-177 236

[20] Wagner F and Stroth U 1993 Plasma Phys Control Fuston 351321

Carreras B A 1997 IEEE Trans Plasma Sct 251281

[21] Garcia L Dıamond P H Carreras B A and Callen J D 1985 Phys Fluads 28247

[22] Reiter D 1992 J Nucl Mater 196-198 80 
[23] Tsu H Y W et al 1992 Rev Sct Instrum 634608

Hıdalgo C 1995 Plasma Phys Control Fusion 37 A53

[24] Perkins F W etal 1993 Phys Flutds B 5477

Petty C C etal 1995 Phys Plasmas 22342

Umansky and LaBombard B $1999 \mathrm{~J}$ Nucl Mater 266-269 721

[25] Gentle K W 1995 Rev Mod Phys 67809

[26] Gunther K 1990 Contrib Plasma Phys 3051

Gunther K and Carlson A 1994 Contrib Plasma Phys 34484

[27] Weınlıch M and Carlson A 1997 Phys Plasmas 42151

[28] Carlson A, Rohde V, Weınlıch M and ASDEX Upgrade Team $1997 \mathrm{~J}$ Nucl Mater 241-243 722

[29] Hutchinson I H 1987 Phys Fluzds 303777

[30] Chung K-S and Hutchinson I H 1991 Phys Fluzds B 33053

[31] Laframboise J G 1997 J Geophys Res 1022417

[32] Onshı T, Martmez-Sanchez M, Cooke D L and Sanmartın J R 2001 Proc International Conf on Electric Propulsion 2001 (Pasadena, Ca NASA)

[33] Stangeby P C 1989 Plasma Diagnostıcs, Surface Analysts and Interactions vol 2, ed O Auciello and D L Flamm (San Diego Academic)

[34] Sanmartın J R 1993 Nuclear Fusion by Inertial Confinement ed G Velarde, Y Ronen and J M Martınez-Val (Boca Raton Chemical Rubber)

[35] Koo B-W and Hershkowitz N 1999 J Appl Phys 86121 